\title{
OPEN Neonicotinoids can cause arrested pupal ecdysis in Lepidoptera
}

\author{
Niranjana Krishnan ${ }^{1,2 \bowtie}$, Russell A. Jurenka ${ }^{1}$ \& Steven P. Bradbury ${ }^{1,2,3}$
}

Recently, we reported a novel mode of action in monarch butterfly (Danaus plexippus) larvae exposed to neonicotinoid insecticides: arrest in pupal ecdysis following successful larval ecdysis. In this paper, we explore arrested pupal ecdysis in greater detail and propose adverse outcome pathways to explain how neonicotinoids cause this effect. Using imidacloprid as a model compound, we determined that final-instar monarchs, corn earworms (Helicoverpa zea), and wax moths (Galleria mellonella) showed high susceptibility to arrested pupal ecdysis while painted ladies (Vanessa cardui) and red admirals (Vanessa atalanta) showed low susceptibility. Fall armyworms (Spodoptera frugiperda) and European corn borers (Ostrinia nubilalis) were recalcitrant. All larvae with arrested ecdysis developed pupal cuticle, but with incomplete shedding of larval cuticle and unexpanded pupal appendages; corn earworm larvae successfully developed into adults with unexpanded appendages. Delayed initiation of pupal ecdysis was also observed with treated larvae. Imidacloprid exposure was required at least $26 \mathrm{~h}$ prior to pupal ecdysis to disrupt the molt. These observations suggest neonicotinoids may disrupt the function of crustacean cardioactive peptide (CCAP) neurons, either by directly acting on their nicotinic acetylcholine receptors or by acting on receptors of inhibitory neurons that regulate CCAP activity.

Neonicotinoids are among the most widely used insecticides in the world. In the United States, nearly all corn hectares and the majority of soybean and cotton hectares are planted with neonicotinoid-treated seeds, which accounts for over $80 \%$ of their total use ${ }^{1}$. In addition, nearly $680,000 \mathrm{~kg}$ of imidacloprid, thiamethoxam, and clothianidin, the three most commonly used neonicotinoids, are applied as spray, soil drench, or injection (soil/ tree) formulations in agricultural land, non-crop land, and urban areas ${ }^{2,3}$. Not surprisingly, studies have reported potential neonicotinoid exposure to target and non-target insect species, including Lepidoptera larvae $\mathrm{e}^{4-8}$.

In insects, neonicotinoids exert their neurotoxic effects by binding to the $\alpha 4 \beta 2$ subunits of the nicotinic acetylcholine receptors ( $\mathrm{AAChR}$ ) in the central nervous system. At toxic doses, receptor binding results in neuronal overstimulation, paralysis, and death ${ }^{9}$. Adverse outcome pathways (AOPs), which are conceptual frameworks that link a molecular initiating event to an adverse outcome ${ }^{10}$, have been proposed for bees at sublethal doses. In the honey bee, Apis mellifera, LaLone et al. ${ }^{11}$ proposed that nAChRs could be desensitized if exposed to prolonged but relatively low neonicotinoid doses. In addition, sublethal exposures are proposed to cause mitochondrial dysfunction, leading to alteration of $\mathrm{Ca}^{2+}$-calmodulin activated signal transduction. Altered $\mathrm{Ca}^{2+}$ transduction could, in turn, prevent translation of proteins involved in long-term memory and thereby cause abnormal foraging behavior that eventually leads to colony failure ${ }^{11}$. Camp and Lehmann ${ }^{12}$ proposed that neonicotinoids could exert similar cellular effects in bumble bees (Bombus terrestris and Bombus impatiens).

In the present paper, we report a novel effect of neonicotinoids on the development of butterflies and moths and propose associated AOPs. We characterize a unique adverse outcome, termed arrested pupal ecdysis (AE), which was first reported following neonicotinoid exposure to final instars of the monarch butterfly (Danaus plexippus; Nymphalidae). In the monarch, AE is characterized by a failure to complete the pupal ecdysis process; the pre-ecdysis and initial ecdysis behaviors initiated by ecdysis triggering hormone (ETH) and eclosion hormone $(\mathrm{EH})^{13}$ are not affected ${ }^{14}$. The final instar that exhibits AE begin shedding their old larval cuticle and trachea from the abdomen but are unable to shed the larval cuticle from the head and ventral thorax. The appendages (antennae, proboscis, wings, and legs) fail to expand into the normal position of an obtect pupae. Larvae died during pupation following topical and dietary exposure to imidacloprid, clothianidin, and thiamethoxam without prior signs of intoxication ${ }^{14,15}$. This disruption in the pupal molt occurred at environmentally relevant doses 10 to 100 -fold lower than doses that caused larval mortality due to neuronal overstimulation and paralysis ${ }^{14,15}$. Larval-to-larval molts were not disrupted at doses that caused AE.

These observations suggest that sublethal neonicotinoid doses (i.e., doses not causing CNS overstimulation) are disrupting neuroendocrine signaling during pupal ecdysis through a novel toxicity pathway. The roles of

${ }^{1}$ Department of Entomology, lowa State University, Ames, IA, USA. ${ }^{2}$ Toxicology Program, lowa State University, Ames, IA, USA. ${ }^{3}$ Department of Natural Resource Ecology and Management, lowa State University, Ames, IA, USA. ${ }^{\circledR}$ email: nkrish@iastate.edu 
Fall armyworm



Wax moth

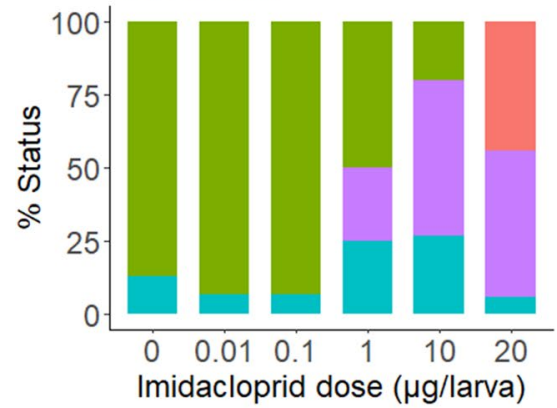

Monarch butterfly

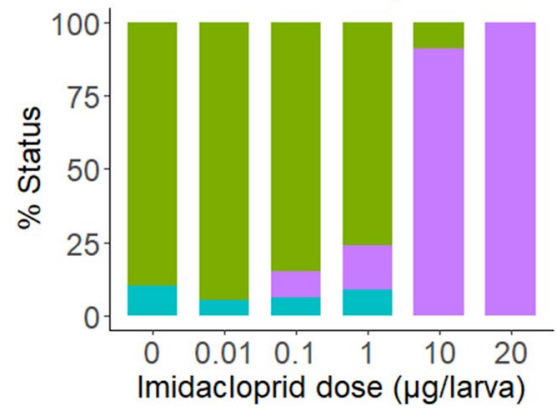

European corn borer

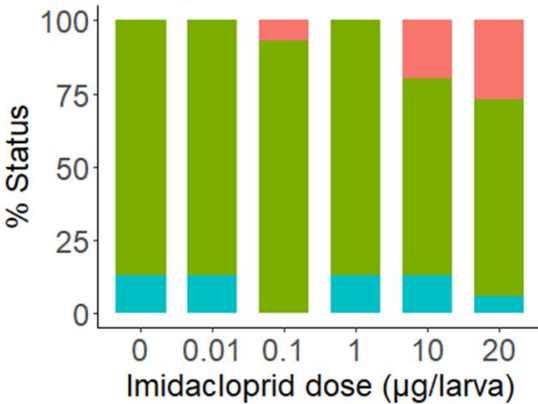

Painted ladies

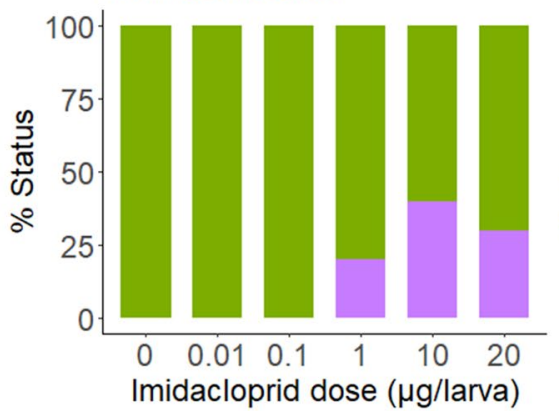

Red admirals



Corn earworm

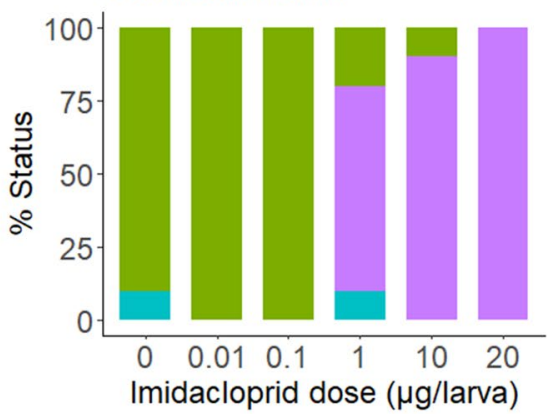

Larval mortality

Pupation with adult eclosion

Pupation with no adult eclosion

AE larvae

Figure 1. Stacked barplots depicting percent of imidacloprid-treated final instars that had larval mortality, pupated with adult eclosion, pupated without adult eclosion, and arrested pupal ecdysis (AE). Number of larvae treated per dose: 10 for fall armyworms, red admirals, painted ladies, and corn earworms, 15 for European corn borers and wax moths, and for monarch butterflies $22-33$ at $0-10 \mu \mathrm{g}$ doses and 10 at the $20 \mu \mathrm{g}$ dose.

neuroendocrine hormones in regulation of pupal ecdysis have been studied in detail. The roles of specific neurotransmitters, however, have not been addressed to date, although several research groups note the need for further investigation ${ }^{13,16}$. Elucidation of neonicotinoid-induced AE symptomology in multiple Lepidoptera and development of hypothesis-based AOPs provides a framework to design future experiments to improve understanding of acetylcholine signaling in regulation of pupal ecdysis. In addition, further characterization of $\mathrm{AE}$ symptomology can support more informed neonicotinoid risk assessments for non-target lepidopteran species and provide new insights on neonicotinoid efficacy against late instars of pest species.

To determine if monarch larvae are unique in displaying AE, we treated six other lepidopteran larvae, belonging to Nymphalidae, Noctuidae, Crambidae, and Pyralidae, with imidacloprid. As not all species were susceptible to AE, we subsequently analyzed concentrations of imidacloprid and its metabolites in an AE-sensitive and an AE-insensitive species over time to determine if the latter metabolized and excreted larger quantities of imidacloprid prior to pupal ecdysis. Results from this experiment also shed light on the range of internal imidacloprid doses needed to elicit AE. By treating larvae with imidacloprid at time points before and after head capsule slippage (HCS), we identified when final instars are susceptible to AE. Finally, to document the phenotypic events resulting in $\mathrm{AE}$, the pupal ecdysis process was documented in fine temporal detail with control and imidaclopridtreated larvae. To formulate proposed AOPs for imidacloprid-induced AE, results from this series of experiments were interpreted in light of literature addressing neuroendocrine control of pupal ecdysis.

\section{Results}

Species' susceptibility to AE. AE was observed in monarchs, corn earworms, wax moths, painted ladies, and red admirals that were topically treated with 1 to $20 \mu \mathrm{g}$ imidacloprid (Fig. 1). Fall armyworms and European corn borers were recalcitrant to $\mathrm{AE}$ even following a $100 \mu \mathrm{g}$ imidacloprid treatment (Table S1). A positive 

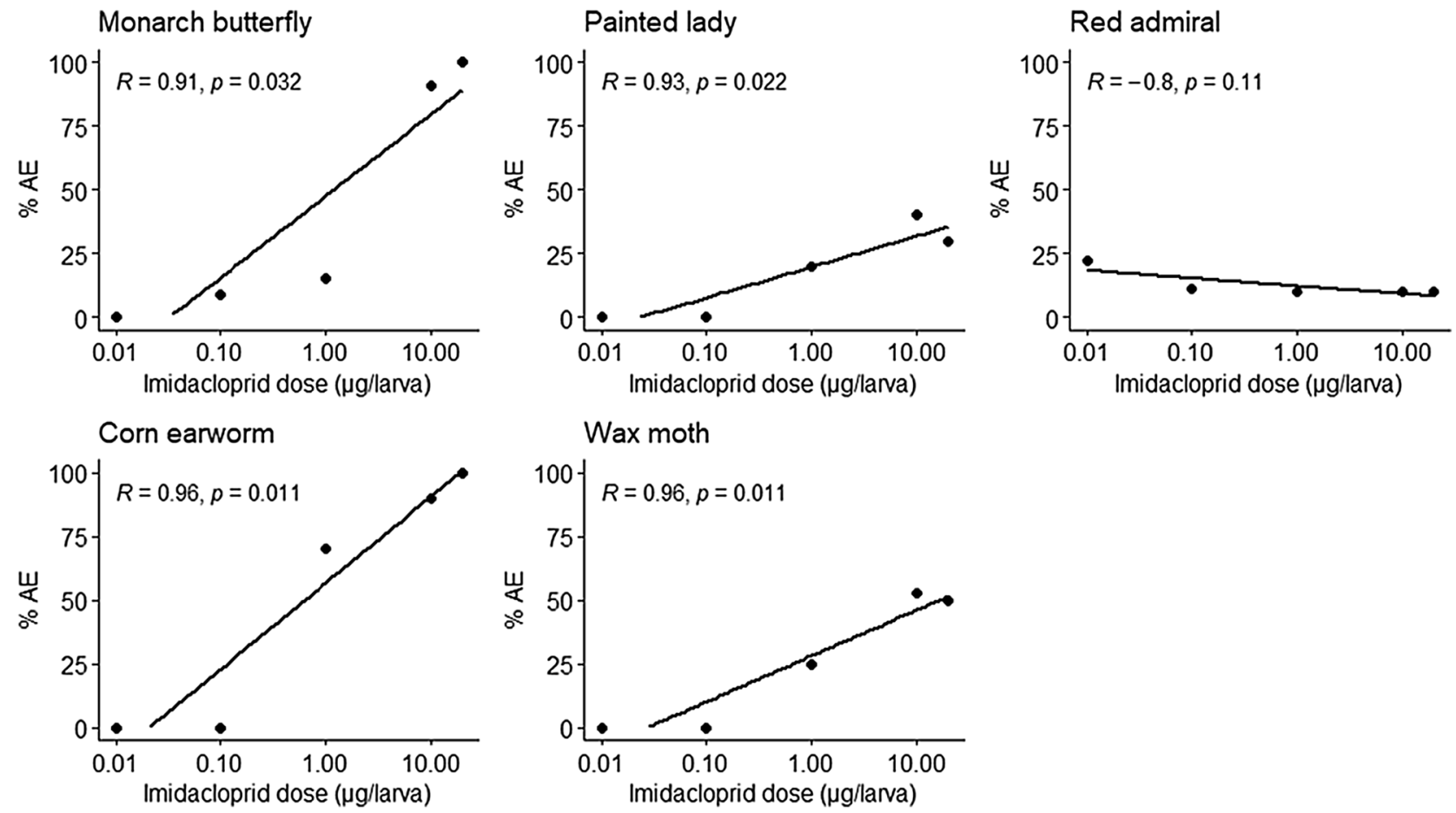

Figure 2. Correlation between imidacloprid dose ( $\mu \mathrm{g} / \mathrm{larva})$ and percent arrested pupal ecdysis (AE) in monarch butterfly $(n=152)$, painted lady $(n=60)$, red admiral $(n=60)$, corn earworm $(n=60)$, and wax moth $(n=92)$ final instars. Pearson's correlation coefficient was used to calculate $\mathrm{R}$ and significance value for each species. Note that the $\mathrm{x}$-axis is on a logarithmic scale.

correlation $(p \leq 0.032)$ between dose and percent AE was seen in all susceptible species except red admirals (Fig. 2). Removal of larval cuticle from AE butterfly larvae and a closer examination of AE moth larvae showed incomplete shedding of larval trachea, a partially shed larval cuticle at the posterior end of the abdomen, a partially complete pupal case (partially complete in that larval cuticle of the head and legs could not be removed to determine if there was an underlying pupal cuticle), and untanned cuticle on the ventral side where the pupal wing cuticle would normally expand (Figs. 3 and S1).

In monarchs, $\mathrm{AE}$ was observed in $100 \%$ of larvae that did not undergo complete pupation at doses $\leq 20 \mu \mathrm{g}$ (Fig. 1). Over $90 \%$ of larvae exposed to 10 and $20 \mu \mathrm{g}$ imidacloprid exhibited AE. At higher doses, mortality mostly occurred through imidacloprid's primary mode of action (i.e., overstimulation of the CNS) (Table S1), which included cessation of feeding, paralysis, and bleeding symptoms. In painted ladies and red admirals, 50 to $100 \%$ of treated larvae that failed to pupate exhibited AE; however, the percent AE at any dose did not exceed $40 \%$ (Fig. 1). For all three butterfly species, larvae expressing AE either died following emergence of the pupal cuticle on the thorax or at the "J" stage, often accompanied by external bleeding. The moth species that expressed $\mathrm{AE}$ had a visible pupal cuticle on the abdomen and the dorsal and posterior sides of the thorax. In wax moths, all larvae that failed to pupate at doses up to $10 \mu \mathrm{g}$ imidacloprid had AE (53\% of treated larvae), while larvae treated with the $20 \mu \mathrm{g}$ dose either exhibited AE or died through imidacloprid's primary mode of action. Four of the 10 AE larvae we dissected initiated, but did not complete, adult development. In corn earworms, all treated larvae that did not successfully pupate had AE, with the 10 and $20 \mu \mathrm{g}$ imidacloprid doses producing AE in 90 and $100 \%$ of larvae, respectively (Fig. 1). Of note, AE often did not lead to immediate mortality in corn earworms. Instead, adults developed within the unshed larval and pupal cuticles. Removal of the old cuticles approximately two weeks after treatment revealed a completely formed adult except the appendages (proboscis, antennae, wings, and legs) were not expanded (Fig. 4).

Based on observations taken every $24 \mathrm{~h}$, there were no differences between control and treated larvae in the number of days it took to initiate pupal ecdysis following treatment ( $p>0.27$ for all species; see Table S2). In all species, doses that produced at least two pupae had adult eclosion rates ranging from 61 to $100 \%$ (Fig. 1).

Imidacloprid toxicokinetics in AE-susceptible and unsusceptible species. Sixth instar fall armyworm (an AE-unsusceptible species) that were topically treated with $20 \mu \mathrm{g}$ imidacloprid and collected within five minutes of treatment contained a mean $( \pm$ SD) of $59( \pm 11) \mu \mathrm{g} / \mathrm{g}$ imidacloprid (Fig. 5); two of the four larval samples also had detectable 5-hydroxy imidacloprid below the limit of quantification $(<0.02 \mu \mathrm{g} / \mathrm{g}$; Table S3). Larvae collected $4 \mathrm{~h}$ after treatment had a similar imidacloprid concentration $(56 \pm 23 \mu \mathrm{g} / \mathrm{g})$ and three of the five samples had one or both imidacloprid metabolites. Larvae collected $24 \mathrm{~h}$ after treatment had four-fold lower imidacloprid concentrations $(13 \pm 7.9 \mu \mathrm{g} / \mathrm{g})$ and four of the five samples contained one or both metabolites. The parent imidacloprid concentration in larvae at $24 \mathrm{~h}$ was significantly lower than at $0 \mathrm{~h}$ ( $\mathrm{t}-$ ratio: -5.135 ; $p=0.0003$; Fig. 5). Pupation occurred approximately four days after treatment and only two of the five pupal 

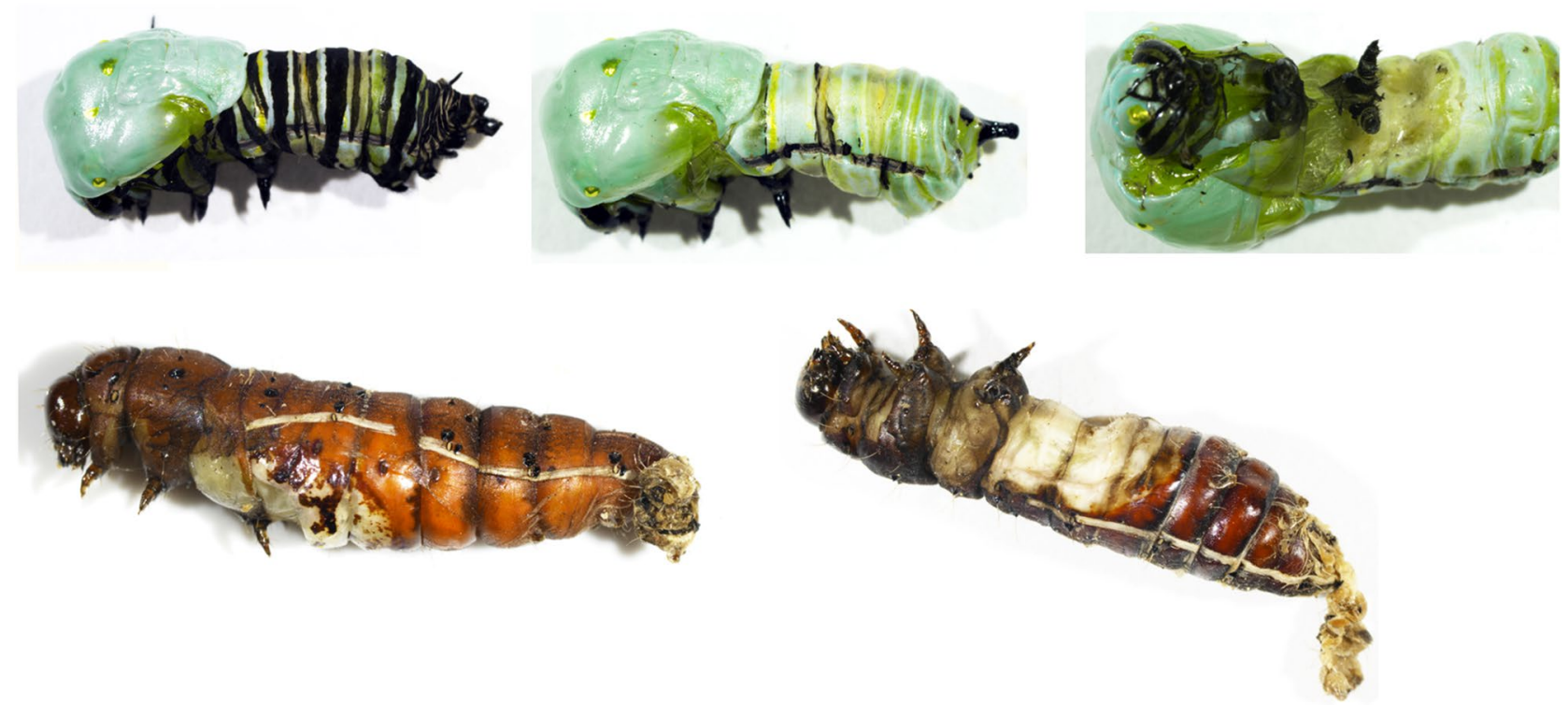

Figure 3. Arrested pupal ecdysis in representative fifth instar monarch butterfly (top row) and sixth instar corn earworm (bottom row) treated with $20 \mu \mathrm{g}$ imidacloprid. Careful removal of old larval cuticle from monarchs (top row middle and right) and an examination of corn earworms showed complete pupal cuticle on the dorsal and posterior side and partially shed tracheal cuticle. In both species, appendages had not expanded and the ventral side of the first abdominal segments were not sclerotized.

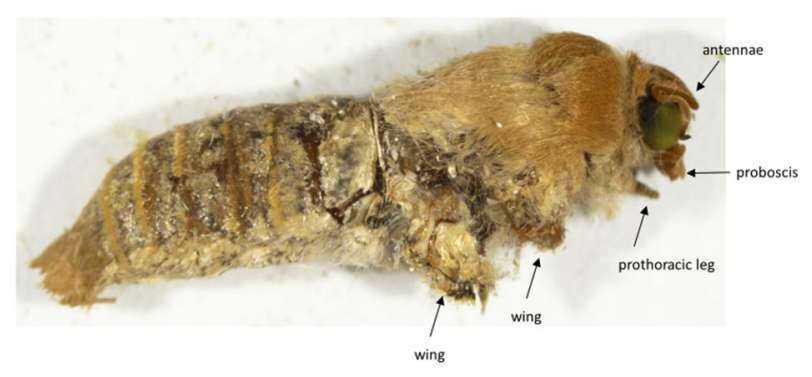

Figure 4. Corn earworm larvae that exhibited arrested pupal ecdysis were allowed to develop as adults. Careful removal of larval and pupal cuticle after adult development shows an adult with appendages (labeled) that did not expand.

samples had detectable concentrations of imidacloprid; in both samples the concentrations were lower than the limit of quantification. Neither of the metabolites were detected in the pupal samples.

Fifth instar monarch butterflies that were topically treated with $20 \mu \mathrm{g}$ imidacloprid and collected within $5 \mathrm{~min}$ of treatment contained a mean of $24( \pm 10) \mu \mathrm{g} / \mathrm{g}$ imidacloprid (Fig. 5). Fifth instars collected $24 \mathrm{~h}$ (larval stage) and $48 \mathrm{~h}$ (AE stage) after treatment contained similar imidacloprid concentrations $(31 \pm 12$ and $20 \pm 3.4 \mu \mathrm{g} / \mathrm{g}$, respectively). The two imidacloprid metabolites were not detected in any of the monarch samples (Table S3). No differences between parent imidacloprid concentrations were found in larvae collected at the different time points post exposure $(\mathrm{F}=1.8496 ; p=0.1994)$.

Effect of imidacloprid at various times prior to pupation. Following topical treatment with $20 \mu \mathrm{g}$ imidacloprid, sixth instar corn earworms treated prior to HCS (ca. 3 days before pupation) had the highest level of AE (90\%) (Fig. 6A). Larvae treated just after HCS (ca. 2 days before pupation) had 75\% AE. Larvae that were treated ca. $12 \mathrm{~h}$ (ca. 1 day before pupation) and $23 \mathrm{~h}$ (ca. 0.5 day before pupation) post HCS, had 20 and $8 \% \mathrm{AE}$, respectively, with the remaining larvae successfully undergoing pupal ecdysis. A significant correlation between day of larval treatment and percent AE was observed ( $p=0.028$; Fig. $6 \mathrm{~B})$. Nearly $90 \%$ of the AE larvae survived and developed to the adult stage and a third of these attempted to emerge but only their abdomen lost the pupal cuticle (data not shown). Of those larvae that successfully pupated, a small percentage had wrinkled appendages, loss of hemolymph, and bloated cuticle around the wings (Figure S2A). Of all the larvae that successfully pupated, only $23 \%(n=5)$ emerged normally, though their eclosion was delayed by one to two days with respect to controls. The remaining pupae either did not emerge or emerged with deformed and uninflated wings (Figs. S2B and 6A). 

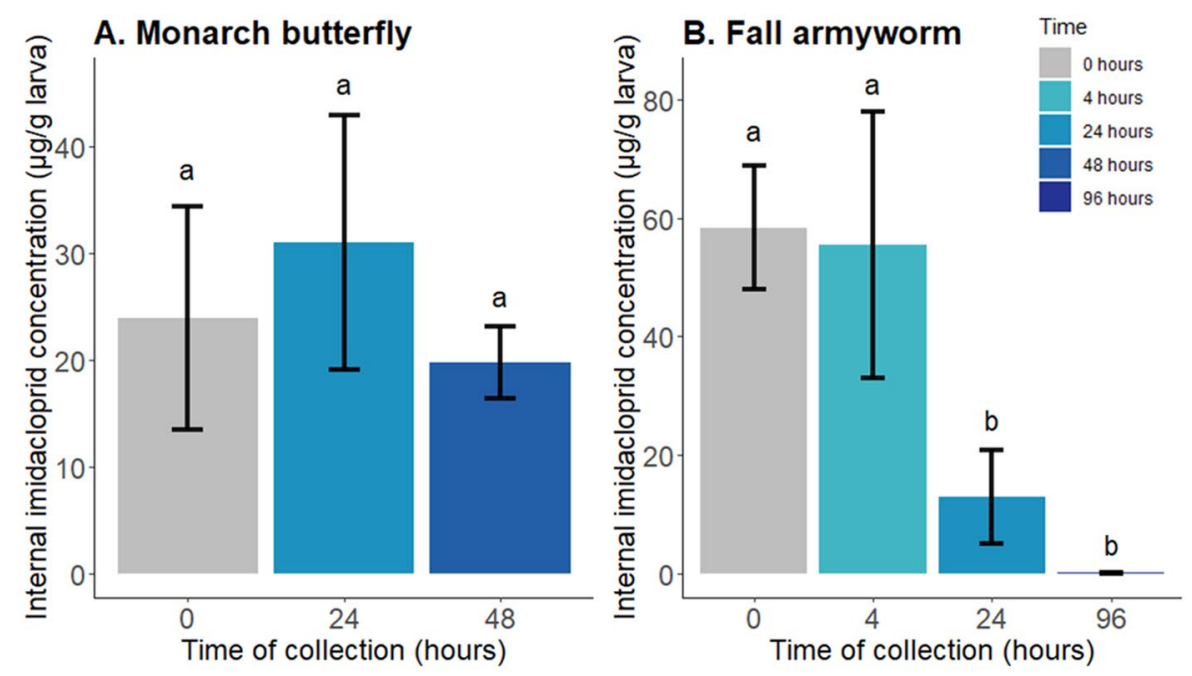

Figure 5. Mean internal concentrations ( \pm standard deviation) of imidacloprid in monarch butterfly (A) and fall armyworm (B) final instars. Larvae were collected at the following time points after topical treatment with $20 \mu \mathrm{g}$ imidacloprid : $0 \mathrm{~h}$ (both species; larvae), $4 \mathrm{~h}$ (fall armyworm; larvae), $24 \mathrm{~h}$ (both species; larvae), $48 \mathrm{~h}$ (monarch butterfly in arrested pupal ecdysis), and $96 \mathrm{~h}$ (fall armyworm as pupae). For each species, different lower-case letters above bars denote significant differences $(p<0.05)$ between control and treated larvae using Dunnett's test for multiple comparisons.

A.

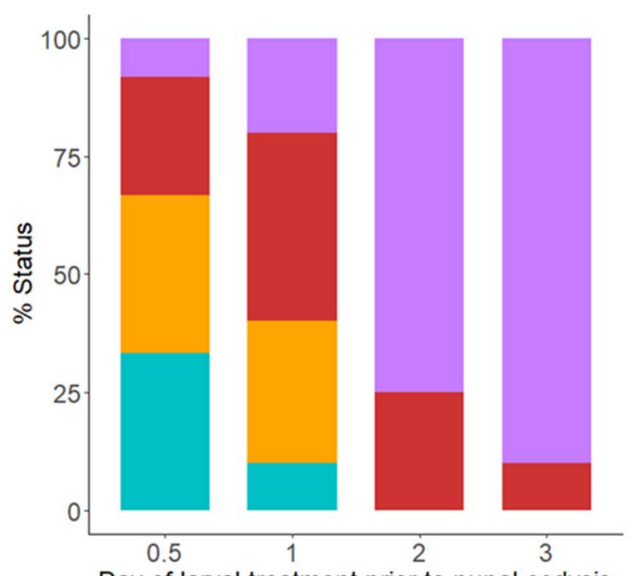

Day of larval treatment prior to pupal ecdysis
B.



AE larvae
Pupation with adult eclosion and deformed wings
Pupation with adult eclosion and normal wings
Pupation with no adult eclosion

Figure 6. Corn earworm larvae were treated topically with $20 \mu \mathrm{g}$ imidacloprid at four time points prior to pupal ecdysis. (A) Stacked barplot depicting the percent of imidacloprid-treated final instar corn earworms that had arrested pupal ecdysis (AE), pupation with no adult eclosion, pupation with adult eclosion and deformed wings, and pupation with adult eclosion and normal wings. (B) Correlation between day of treatment and percent AE. Pearson's correlation coefficient was used to calculate $\mathrm{R}$ and significance. Eight to 12 larvae were treated per time point. All of the control larvae ( $n=4$ to 6 per time point) had successful pupation with normal adult eclosion.

While monarch larvae also showed high rates of AE when topical treatments were applied 2-3 days before pupation $^{14}$, the pattern of AE expression following dietary exposure differed. Final-instar monarchs that were 2-3 days from pupation and fed $0.5 \mu \mathrm{g}$ of imidacloprid/g leaf for $24 \mathrm{~h}$, followed by 24 -h feeding on untreated leaves, pupated normally (Table S4). However, final-instar monarchs that were 1-2 days from pupation and only provided leaves treated with imidacloprid for $24 \mathrm{~h}$ expressed AE. These differences in AE expression based on topical and dietary exposure routes and timing were also observed in previous work ${ }^{14,15}$. To help explain these 


\begin{tabular}{|l|l|l|l|l|}
\hline Treatment $(\mathbf{n})$ & Percentage & $\begin{array}{l}\text { Mean }( \pm \text { SD) hours to start of HCS } \\
{[\mathbf{A}]^{\mathbf{b}}}\end{array}$ & $\begin{array}{l}\text { Mean }( \pm \text { SD) hours to start of } \\
\text { pupation/AE }[\mathbf{B}]^{\mathbf{b}}\end{array}$ & $\begin{array}{l}\text { Mean }( \pm \text { SD) hours from start of } \\
\text { HCS to start of pupation/AE }[\mathbf{B}-\mathbf{A}]\end{array}$ \\
\hline Acetone $(\mathrm{n}=12$ larvae$)$ & Pupation: $100 \%$ & $42( \pm 3.7)($ range: 39 to $49 \mathrm{~h})$ & $68( \pm 3.7)($ range: 64 to $75 \mathrm{~h})$ & $26( \pm 1.7)($ range: 22 to $29 \mathrm{~h})$ \\
\hline Imidacloprid $(\mathrm{n}=12$ larvae $)$ & AE: $100 \%$ & $42( \pm 3.4)($ range: 39 to $49 \mathrm{~h})$ & $73( \pm 7.5)($ range: 68 to $94 \mathrm{~h})$ & $31( \pm 6.4)($ range: 26 to $49 \mathrm{~h})$ \\
\hline
\end{tabular}

Table 1. Time to head capsule slippage and pupation or arrested pupal ecdysis for corn earworm sixth instars topically treated with acetone or $20 \mu \mathrm{g}$ imidacloprid ${ }^{\mathrm{a}}$. HCS head capsule slippage, AE arrested (pupal) ecdysis, $S D$ standard deviation. ${ }^{a}$ Larvae were approximately 2 -day old sixth instars at the time of treatment. ${ }^{\text {b Hours to }}$ HCS and pupation/AE were calculated from time of treatment.

differences, imidacloprid concentrations within monarch samples were measured. Pupal samples from larvae that fed on treated leaves for $24 \mathrm{~h}$ followed by feeding on untreated leaves for $24 \mathrm{~h}$ had imidacloprid concentrations below the level of quantification $(>0.02 \mathrm{ug} / \mathrm{g})$. Larvae with $\mathrm{AE}$ that were not provided untreated leaves had a mean $( \pm \mathrm{SD})$ of $0.14( \pm 0.06) \mu \mathrm{g} / \mathrm{g}$ imidacloprid (Table S4). These results suggest differences in AE susceptibility between topical and dietary exposure may be attributed to faster rates of imidacloprid excretion following dietary exposure.

Observational details on pupation in corn earworms. On average, both acetone-treated and imidacloprid-treated sixth instar corn earworms initiated HCS $42 \mathrm{~h}$ after exposure. Pupal ecdysis was initiated at a mean $( \pm S D)$ of $68( \pm 3.7)$ and $73( \pm 7.5)$ hours post exposure, respectively (Table 1$)$. Following HCS, the imidacloprid-treated larvae initiated the ecdysis motor process approximately $5 \mathrm{~h}$ later than the controls, i.e., 31 $( \pm 6.4)$ versus $26( \pm 1.7)$ hours for controls. This difference was statistically significant at $\alpha=0.05$ level $(t=-2.859$; $p=0.0139)$.

Acetone-treated corn earworm larvae took ca. $10 \mathrm{~min}$ to complete pupal ecdysis, from initiation of tracheal shedding through complete shedding of larval cuticle. After ca. 1 to $2 \mathrm{~min}$ following initiation of the posterior abdomen larval cuticle and tracheal shedding, the larval cuticle at the dorsal thorax split along the ecdysial line and the pupal cuticle started to emerge. Approximately one minute later, the larvae turned over, exposing the ventral side and began shedding its larval cuticle from the anterior. Expansion of the pupal appendages began just before ecdysis and continued during and after ecdysis. Immediately following ecdysis, the wing length was ca. $5 \mathrm{~mm}$ and expanded to $10 \mathrm{~mm}$ over the next $15 \mathrm{~min}$ prior to cuticle hardening and tanning.

Imidacloprid-treated corn earworm larvae behaved as previously described; they initiated, but did not complete, abdominal cuticle and tracheal shedding. While the larval cuticle at the dorsal thorax did not split in most larvae, some did split the dorsal thoracic cuticle along ecdysial lines ca. $5 \mathrm{~min}$ after initiation of abdominal cuticle and tracheal shedding. None of the imidacloprid-treated larvae progressed beyond this point, i.e., they did not turnover to expose the ventral side and begin shedding their larval cuticle. These larvae also did not expand their appendages. For two AE corn earworm larvae that continued the ecdysis process past the abdominal cuticle and tracheal shedding, the larval cuticle was removed from the abdomen but the thoracic and head larval cuticle remained. After approximately $30 \mathrm{~min}$, we carefully removed the larval cuticle from the thorax and head. The larval cuticle over the legs and head could be successfully pulled off and revealed a complete pupal cuticle except the appendages were not expanded (Figure S3).

\section{Discussion}

In the following sections, we interpret and integrate results from our experiments to gain a better understanding of AE symptomology and differences in interspecies susceptibility. Based on our observations and review of the insect ecdysis literature, we propose two AOPs for neonicotinoid-induced AE. We do note AE-like effects were reported by Bargar et al. ${ }^{17}$ when they chronically exposed monarch larvae to clothianidin-treated milkweed plants, and by Heneberg et al. ${ }^{18}$ when they treated crabronid wasp prepupae with neonicotinoids. While these studies are broadly concordant with our observations, they do not provide detailed descriptions of the symptoms or their time course. There are currently no published papers that attempt to elucidate the potential mechanism(s) by which sublethal doses of neonicotinoid insecticides could cause AE.

Conservation of neonicotinoid-induced AE across Lepidoptera. High rates (50 to 100\%) of AE were observed in monarchs (Nymphalidae), corn earworms (Noctuidae), and wax moths (Pyralidae). Painted ladies and red admirals (both Nymphalidae) had lower rates (10 to 40\%) of AE. The larval cuticle of these two species are densely covered with hairs and it is possible imidacloprid was not completely absorbed. However, even with dietary exposures, painted ladies are approximately 70 -fold less sensitive to the neonicotinoid clothianidin when compared to monarch $s^{7,15}$ suggesting additional toxicokinetic or toxicodynamic factors contribute to painted ladies relatively low sensitivity to neonicotinoids. European corn borers (Crambidae) and fall armyworms (Noctuidae) were recalcitrant to AE. These findings suggest susceptibility to AE, and corresponding low toxicity of imidacloprid, may not be related to lepidopteran phylogenetic similarity. As both non-target (butterfly) and pest (moth) species show susceptibility to AE, these findings have implications in lepidopteran non-target conservation in agricultural landscapes and in lepidopteran pest management.

To assess if interspecies variability in AE sensitivity could be due, in part, to differences in toxicokinetics, we topically treated monarch and fall armyworm larvae and quantified internal concentrations of imidacloprid and two of its toxic metabolites. In monarchs, internal imidacloprid concentrations remained stable over $48 \mathrm{~h}$ and 
neither of the metabolites were detected in any samples. In fall armyworms, the internal imidacloprid concentration fell rapidly; 24-h larval samples had four-fold lower imidacloprid concentrations than the 0 -h samples and, by $96-\mathrm{h}$, imidacloprid was undetectable. Additionally, imidacloprid metabolites were detected at the 0,4 , and 24-h sampling points. While these data show fall armyworms metabolize and excrete imidacloprid more efficiently than monarchs, examination of insecticide uptake and distribution in the CNS and endocrine-active tissues over time is needed to fully assess the extent of toxicokinetic differences between the species. We also cannot rule out toxicodynamic considerations that could explain the differences in species susceptibility, e.g., interspecies differences in imidacloprid binding potential to nAChRs.

Rationale for a novel mode of action. There are several observations that suggest imidacloprid-induced AE occurs through a novel toxicity pathway. When imidacloprid acts through its primary mode of action, larvae show signs of CNS poisoning (cessation of feeding, paralysis, bleeding) within $24 \mathrm{~h}$ and die soon thereafter ${ }^{14}$. Early instars are more sensitive to the primary mode of action ${ }^{14}$, possibly due to their smaller body weights. In contrast, with AE we observe no neurotoxic symptoms and instead observe delayed mortality, greater sensitivity of final instars, and a narrow temporal window of susceptibility at doses below the threshold that elicit symptoms associated with the CNS intoxication.

Delayed mortality with no neurotoxic symptoms. Monarchs, corn earworms, painted ladies, and red admirals that exhibited $\mathrm{AE}$ at doses $\leq 20 \mu \mathrm{g}$ and wax moths that exhibited $\mathrm{AE}$ at doses $\leq 10 \mu \mathrm{g}$ showed no detectable symptoms prior to ecdysis. Most AE larvae had initiated pupal ecdysis 2 to 6 days after treatment, similar to controls. Fifth-instar corn earworms topically treated with $20 \mu \mathrm{g}$ imidacloprid molted to the sixth instar with no symptoms and then exhibited AE (Figure S4) 6 to 8 days after treatment. Additionally, corn earworm larvae that exhibited AE did not die immediately and developed adult features.

Greater sensitivity of final instars. Previously we had published dietary toxicity data for monarch second, third, and early fifth instars provided imidacloprid-treated milkweed leaves for 24 to $48 \mathrm{~h}^{14,15}$. A dietary exposure equivalent to 1.5 to $7.0 \mu \mathrm{g}$ of imidacloprid/g larva caused 0 to $5 \%$ mortality, with none attributed to AE. However, when late fifth instars were provided an imidacloprid dose of $0.76 \mu \mathrm{g} / \mathrm{g}, 100 \%$ mortality occurred, all through $\mathrm{AE}^{15}$. With topical exposures, a $9.1 \mu \mathrm{g} / \mathrm{g}$ imidacloprid dose caused $90 \%$ mortality in fifth instars; doses that were two $(17 \mu \mathrm{g} / \mathrm{g})$ and six $(56 \mu \mathrm{g} / \mathrm{g})$ times greater were needed to cause similar levels of mortality through CNS intoxication in first and third instar larvae, respectively ${ }^{14}$.

AE effects at low doses. In monarch fifth instars, the average doses that caused over $90 \%$ mortality through $\mathrm{AE}$ ranged from 0.76 (dietary exposure) to 9.1 (topical exposure) $\mu \mathrm{g} / \mathrm{g}$ larvae; doses of 124 (dietary exposure) and 91 (topical exposure) $\mu \mathrm{g} / \mathrm{g}$ are needed to cause similar rates of mortality through imidacloprid's primary mode of action ${ }^{14,15}$. These results indicate that the imidacloprid doses that cause AE in monarchs are 10 (topical exposure) to 160 (dietary exposure) fold lower than doses that cause larval mortality through the primary neurotoxic mode of action.

Narrow temporal window of susceptibility. AE symptomology is specific to larval to pupal ecdysis; in both monarchs and corn earworms, larval to larval ecdysis was unaffected ${ }^{14,15}$ (Figure S4). Further, in sixth instar corn earworm larvae, we observed high rates of $\mathrm{AE}$ (75 to 100\%) when topical imidacloprid exposures occurred ca. 1 day before HCS or immediately after HCS. If larvae were treated 0.5 or 1 day after HCS, the vast majority of larvae (80 to 92\%) formed complete pupae (Fig. 6). Of note, HCS occurs after decline of the second ecdysteroid peak, which leads to the release of ETH peptides that initiate the ecdysis process ${ }^{13}$. While additional toxicokinetic data that provide a more refined time course of insecticide absorption and distribution are needed, the findings to date nevertheless suggest that neonicotinoids disrupt pupal ecdysis within a specific and relatively small window of time.

Interpretation of AE symptomology. As described previously, AE typically does not lead to immediate mortality in corn earworms; consequently, an analysis of these larvae provided useful insights on the time course of key events. We have observed the following:

1. AE occurred after initiation of pupal ecdysis and was characterized by incomplete shedding of trachea and larval cuticle.

2. AE larvae could form complete pupae and adults (i.e., development was not affected); however, the pupal and adult appendages were unexpanded/uninflated.

3. High rates of AE were observed if topical imidacloprid exposure occurred just prior to pupal HCS.

4. Imidacloprid did not disrupt larval to larval ecdysis.

5. Imidacloprid delayed initiation of pupal ecdysis.

6. Imidacloprid-treated larvae that successfully pupated had delayed adult eclosion.

Based on these observations, we propose neonicotinoids are interfering with the function of crustacean cardioactive peptide (CCAP) neurons. Neurons containing CCAP help maintain ecdysis, which is initiated by ETH and EH. In fruit flies (Drosophila melanogaster), CCAP neurons are necessary for pupal leg and wing expansion, timely eclosion, and adult wing inflation ${ }^{19,20}$. In the red flour beetle, Tribolium castaneum, knockdown of the gene encoding CCAP resulted in arrested adult ecdysis, but the pupae had normal pre-ecdysis behaviors ${ }^{21}$. CCAP 




Figure 7. Proposed adverse outcome pathways that elucidate how neonicotinoid treatment could lead to arrested pupal ecdysis (AE). MIE molecular initiating event, $K E$ key event, $A O$ adverse outcome.

neurons are also needed for successful pupal, but not larval, ecdysis. Failed pupal ecdysis following ablation of CCAP neurons is characterized by incomplete shedding of larval trachea and failure to extend appendages ${ }^{19,22}$. In the hemimetabolous insect Rhodnius prolixus, knockdown of CCAP has also been reported to delay initiation of the ecdysis motor process ${ }^{23}$.

Different subsets of CCAP neurons release CCAP, myoinhibitory peptides (MIPs), and bursicon peptides during and after ecdysis ${ }^{13}$. Bursicon is responsible for cuticle sclerotization and tanning; however, the pupal and adult cuticle of neonicotinoid-treated corn earworm larvae have coloration and hardness similar to control cuticle (the unsclerotized cuticle on the ventral side of AE pupae can be attributed to unexpanded appendages). In fruit flies, bursicon subunits are also believed to be necessary for successful pupal ecdysis and adult wing inflation $^{22}$. Of note, just prior to pupal ecdysis in fruit flies, there is an emergence of 12 'late' CCAP neurons that differentiate to express CCAP and bursicon. These neurons alone are sufficient to initiate pupal ecdysis ${ }^{20}$. These 'late' CCAP neurons are necessary for pupal leg extension, while the 'early' CCAP neurons are necessary for adult wing inflation. In total, these observations in fruit flies suggest that in Lepidoptera, the neonicotinoids may be disrupting signaling from both 'early' and 'late' CCAP neurons, including subsets of bursicon-expressing CCAP neurons. Although the CCAP neurons are conserved in arthropods ${ }^{24}$, including Lepidoptera ${ }^{25}$, it is not known if the 'early' and 'late' CCAP neuron functionality is conserved.

Proposed adverse outcome pathways for AE in Lepidopteran. We propose two AOPs that provide testable hypotheses to elucidate the molecular initiating events and resultant key events associated with neonicotinoid-induced AE (Fig. 7). In developing the proposed AOPs, we reviewed AOPs for neonicotinoid-induced effects on honeybee colony death ${ }^{11}$ and an AOP for ecdysone agonists that leads to lethal molting disruption ${ }^{26}$. We also reviewed the literature addressing neuroendocrine pathways that initiate and regulate ecdysis.

Molecular initiating event. Consistent with their primary CNS-based mechanism of action, we propose that $\mathrm{AE}$ is also initiated by neonicotinoid binding to $\mathrm{nAChRs,} \mathrm{but} \mathrm{in} \mathrm{a} \mathrm{distinct} \mathrm{group} \mathrm{of} \mathrm{neurons.} \mathrm{While} \mathrm{the} \mathrm{neoni-}$ cotinoid's primary mode of action involves their agonism of acetylcholine at nAChRs, it is possible AE could be due to interactions at other receptor binding sites. However, we previously reported increased rates of AE in monarch fifth instars topically treated with chlorpyrifos ${ }^{14}$, which is an acetylcholine esterase inhibitor that has the net effect of increasing acetylcholine signaling. To determine if this response could be replicated in another susceptible species, we treated sixth instar corn earworm larvae with chlorpyrifos. Of the ten corn earworms topically treated with $140 \mu \mathrm{g}$ chlorpyrifos, seven exhibited AE (data not shown). These findings suggest that disruption of acetylcholine signaling pathways may be central to AE.

In holometabolous insects, several steps occur sequentially to initiate pupal ecdysis. In moths, following the release of ETH, pre-ETH (PETH), and ETH-associated peptide (ETH-AP) from Inka cells, various neurons are 
activated including the L3, 4 neurons that release diuretic hormones and kinins, VM neurons that release EH, and the CCAP neurons ${ }^{13,19,22}$. In fruit flies, subsets of CCAP neurons possess different receptors including ETHR, EHR, nAChR, muscarinic acetylcholine receptors (mAChR), GABA, and glutamate receptors ${ }^{16,27-29}$. Perturbation of normal ETH, EH, acetylcholine, GABA, and glutamate signaling could interfere with CCAP activation and/or secretion of neuropeptides and cause symptoms similar to the neonicotinoid-induced AE. In this regard, Vömel and Wegener ${ }^{29}$ reported that in fruit flies, the vast majority of CCAP neurons respond to acetylcholine (which binds to $\mathrm{nAChR}$ and $\mathrm{mAChR}$ ) and nicotine (which binds to $\mathrm{nAChR}$ ). Desensitization of response was observed at higher nicotine concentrations. Subsets of CCAP neurons that were inhibited by GABA were stimulated by acetylcholine. In vivo studies indicated nicotine could activate presynaptic inhibitory neurons, which are likely GABA neurons.

Key events. Studies in tobacco hornworm (Manduca sexta) have shown that cGMP production can take place in certain nitric oxide (NO)-sensitive CCAP neurons only during pupal ecdysis ${ }^{30}$. In turn, elevated cGMP can lead to increased excitability of CCAP neurons ${ }^{31}$. Zayas et al. ${ }^{32}$ and Mannai et al. ${ }^{33}$ showed that nicotinic acetylcholine receptors can control cGMP levels by coupling to NO production. While cGMP elevation in CCAP neurons mark the activation of the ecdysis circuitry, release of CCAP neuropeptides and initiation of ecdysis behavior takes place $30 \mathrm{~min}$ later. This delay in release of peptides is likely caused by a descending inhibitory input $^{34,35}$. In fruit flies, GABA neurons are partly responsible for this inhibition (i.e., delayed activation) of CCAP neurons $s^{36}$.

Neonicotinoids could initiate AE through two pathways (Fig. 7). They could directly bind to nAChRs on a subset of CCAP neurons and cause receptor overstimulation and desensitization ${ }^{11}$. Alternatively, neonicotinoids could bind to nAChRs on GABA neurons that inhibit CCAP neurons prior to ecdysis. Continued activation of GABA-releasing neurons could delay or diminish disinhibition of a subset of CCAP neurons. Both these AOPs would have a net effect of reduced cGMP elevation and reduced activation of CCAP neurons. In turn, reduced release of CCAP neuropeptides would attenuate the ecdysis motor process (weaker ecdysis movements and abdominal contractions) and prevent expansion of appendages, consistent with observed AE symptomology and mortality.

\section{Conclusions}

We describe a unique adverse effect caused by neonicotinoid insecticides in butterflies and moths. Specifically, we show that neonicotinoids cause failure of pupal (but not larval) ecdysis at doses below those that cause mortality through CNS neurotoxicity. We propose that neonicotinoids at lower doses, which do not elicit symptomology associated with CNS neurotoxicity, interfere with the release of neuropeptides involved in the ecdysis motor program and expansion of pupal appendages. While the majority of lepidopteran species we studied were susceptible to AE, some species were recalcitrant to the effect, which may, in part, be due to species' differences in neonicotinoid toxicokinetics. Further investigation of the mechanisms through which neonicotinoids induce AE will likely aid in better understanding of the insect molting and metamorphosis processes and help refine neonicotinoid risk assessments for non-target lepidopteran species of conservation concern. We propose two AOPs that hypothesize different molecular initiating events through which neonicotinoids could attenuate signaling from CCAP neurons that are necessary for continuation of the ecdysis motor process and extension of appendages during the larval to pupal molt.

\section{Materials and methods}

Insect rearing. Monarch butterfly and European corn borer (Ostrinia nubilalis; Crambidae) larvae were obtained from colonies established by the U.S. Department of Agriculture in Ames, IA. Monarch larvae were fed on greenhouse-grown tropical milkweed (Asclepias curassavica) leaves ${ }^{14}$, while European corn borer larvae fed on an artificial $\operatorname{diet}^{37-39}$. Larvae of both species were reared in incubators maintained at $26.6{ }^{\circ} \mathrm{C}, 65 \%$ relative humidity, and 16:8 light: dark cycle.

Corn earworm (Helicoverpa zea; Noctuidae) and fall armyworm (Spodoptera frugiperda; Noctuidae) eggs were purchased from Benzon Research, Carlisle, PA, and painted lady (Vanessa cardui; Nymphalidae) eggs were purchased from Carolina Biological Supply Co., Burlington, NC. Larvae were fed ad libitum on an artificial diet (Stonefly Heliothis Diet, Ward's Science) and were reared in an incubator maintained at $26^{\circ} \mathrm{C}$ and $14: 10$ light: dark cycle.

Ovipositing female red admiral butterflies (Vanessa atalanta; Nymphalidae) were collected from common nettle (Urtica dioica) in June 2020 from prairies in Story and Boone counties, IA, USA. Larvae that hatched from eggs laid by the captured females were fed common nettle leaves. Wax moth (Galleria mellonella; Pyralidae) larvae were purchased from a commercial store in Ames, IA, USA. Larvae were fed multigrain baby cereal (Gerber brand) that was mixed with water to achieve consistency of thick peanut butter. Red admirals and wax moths were reared in an incubator at $26.6^{\circ} \mathrm{C}, 80 \%$ relative humidity, and 16:8 light: dark cycle.

Chemicals and insecticide solutions. Analytical grade imidacloprid (IUPAC name: N-[1-[(6-chloropyridin-3-yl)methyl]-4,5-dihydroimidazol-2-yl]nitramide; CAS number: 138261-41-3; Percentage purity: $100 \%$ ) was purchased from Sigma-Aldrich. To prepare insecticide stock solutions for topical and dietary bioassays, certified ACS reagent grade acetone, certified ACS reagent grade dimethylformamide (DMF), and Silwet L-77 were purchased from Fisher Scientific.

For the topical bioassays, 20 and $10 \mathrm{mg} / \mathrm{mL}$ imidacloprid stock solutions were prepared in acetone. Tenfold serial dilutions through $0.01 \mathrm{mg} / \mathrm{mL}$ imidacloprid were made with the $10 \mathrm{mg} / \mathrm{mL}$ solution. For the dietary bioassay, $10 \mathrm{mg}$ of imidacloprid was dissolved in $10 \mathrm{~mL}$ of DMF; the stock solution was diluted ten-fold to $0.1 \mathrm{mg} / \mathrm{mL}$ 
with DMF. This concentration was serial diluted with a suspension of $0.1 \%$ silwet: water to obtain a $0.01 \mathrm{mg} / \mathrm{mL}$ (or $0.01 \mu \mathrm{g} / \mu \mathrm{L}$ ) imidacloprid suspension.

Toxicity bioassays. All bioassays were conducted from January 2020 to January 2021 at the environmental conditions specified in "Insect rearing" section.

Species' susceptibility to AE. Studies were conducted on 1- or 2-day-old final-instar larvae of European corn borers (fifth instar), corn earworms (sixth instar), fall armyworms (sixth instar), painted ladies (fifth instar), red admirals (fifth instar), and wax moths (sixth instar). All larvae were placed in individual rearing containers and randomly assigned a treatment prior to starting a bioassay. One $\mu \mathrm{L}$ of acetone or an imidacloprid-acetone solution was placed on the dorsal prothorax using a $10 \mu \mathrm{L}$ pipette. Five imidacloprid concentrations and an acetone control were used. At least ten larvae were treated in each of the six groups and five control larvae were weighed prior to treatment. Daily observations for mortality were taken until pupation and at adult eclosion. Signs of larval intoxication (e.g., spasms, paralysis) and formation of malformed pupae were recorded. Dissections were performed on a subset of larvae that had AE. Photographs were taken with a Nikon DS-Ri2 digital camera connected to a Nikon SMZ1270 stereo microscope. The results obtained were compared to topical imidacloprid exposures conducted on fifth instar monarch butterfly ${ }^{14,15}$ to provide a more complete description of AE.

Imidacloprid toxicokinetics in AE-susceptible and unsusceptible species. Fifth instar monarch larvae and sixth instar fall armyworm larvae were topically treated with a nominal dose of $20 \mu \mathrm{g}$ imidacloprid, as outlined in previous experiments. Five whole larval and pupal samples, each with a minimum mass of $400 \mathrm{mg}$ for armyworms (two individuals) and $800 \mathrm{mg}$ for monarchs (one individual) were collected at the start of an experiment and at the following time points post-treatment: $4 \mathrm{~h}$ (armyworms), $24 \mathrm{~h}$ (monarchs and armyworms), and after pupation or arrested ecdysis (monarchs and armyworms). All larval/pupal samples were stored individually in plastic containers at $-20^{\circ} \mathrm{C}$ until quantification of imidacloprid and its metabolites.

All samples were analyzed for imidacloprid and its two metabolites, 5-hydroxy imidacloprid and imidacloprid olefin, which are toxic to insects ${ }^{40}$. Samples were individually homogenized using a mortar and pestle. A $0.2 \mathrm{~g}$ portion of the sample was extracted with acetonitrile. Approximately $1 \mathrm{~mL}$ of the extract was transferred to a dispersive solid phase extraction (dSPE) tube containing $150 \mathrm{mg} \mathrm{MgSO} 4,50 \mathrm{mg}$ PSA, and $50 \mathrm{mg}$ C18. Extracts were diluted with 50:50 methanol: water and internal standards (5-OH imidacloprid-d4, 13C, $15 \mathrm{~N}$ imidacloprid olefin, and imidacloprid-pyridine-d4-methylene-d2) were added prior to LC-MS analysis. An injection volume of $2 \mu \mathrm{L}$ was used. A Vanquish Flex LC pump interfaced with a TSQ Altis triple quadrupole mass spectrometer (Thermo Fisher Scientific) were used for the analysis. All insecticides were analyzed in positive electrospray ionization mode. The MS ionization source conditions were as follows: spray voltage $3700 \mathrm{~V}$, sheath gas 30 (Arb), auxiliary gas 6 (Arb), sweep gas 1 (Arb), ion transfer tube temperature $325^{\circ} \mathrm{C}$, and vaporizer temperature $350{ }^{\circ} \mathrm{C}$. MS acquisition was performed in selected reaction monitoring (SRM) mode with argon used as the collision gas. Data analysis was performed using Xcalibur 4.2 software (Thermo Fisher Scientific).

Chromatographic separation was achieved using an AccucoreaQ column $(100 \times 2.1 \mathrm{~mm}, 2.6 \mu \mathrm{m})$. The column compartment temperature was $30^{\circ} \mathrm{C}$. Mobile Phase A was $2 \%$ methanol, $5 \mathrm{mM}$ ammonium formate, and $0.1 \%$ formic acid in water and Mobile Phase B was $2 \%$ water, $5 \mathrm{mM}$ ammonium formate, and $0.1 \%$ formic acid in methanol. The gradient conditions were as follows: start at $0 \% \mathrm{~B}$, linear ramp to $100 \% \mathrm{~B}$ at 8 min, hold at $100 \%$ $\mathrm{B}$ for $2 \mathrm{~min}$, drop to $0 \% \mathrm{~B}$ in half a min, and hold at $0 \% \mathrm{~B}$ for $1.5 \mathrm{~min}$. The flow rate was $0.3 \mathrm{~mL} / \mathrm{min}$ and the total run time of the method was $12 \mathrm{~min}$. For both species, a calibration curve that ranged from 0.02 to $2.5 \mu \mathrm{g} / \mathrm{g}$ was prepared with control larvae. Quality control (QC) samples were prepared in triplicate at $0.3 \mu \mathrm{g} / \mathrm{g}$ and analyzed with experimental samples. All QC samples had a calculated concentration within $15 \%$ of the nominal value. The percent recovery was $99 \%$ for imidacloprid, $94 \%$ for 5-hydroxy imidacloprid, and $95 \%$ for imidacloprid olefin.

Effect of imidacloprid at various times prior to pupation. Sixth-instar corn earworm larvae were topically treated with $1 \mu \mathrm{L}$ of $20 \mu \mathrm{g} / \mu \mathrm{L}$ imidacloprid-acetone solution at four different stages of molting as measured by stemmata pigment movement during head capsule slippage (HCS): (1) stemmata intact (several hours prior to HCS), (2) start of HCS, (3) 8 to $14 \mathrm{~h}$ after HCS (average $12 \mathrm{~h}$ ), and (4) 20 to $24 \mathrm{~h}$ after HCS (average $23 \mathrm{~h}$ ). A minimum of eight larvae were treated per stage. Acetone controls $(n=4$ to 6$)$ at each stage were employed. During the course of the experiments, signs of intoxication, daily mortality until pupation, pupal development, and adult eclosion were recorded. A subset of AE larvae was dissected and observed with the dissecting microscope.

Previously published papers suggested that the developmental window of susceptibility to AE varied between topical and dietary exposure routes ${ }^{14,15}$; this difference could be due to toxicokinetic factors. To evaluate differences in AE expression with the timing and route of exposure, $16 \mathrm{fifth}$ instar monarch larvae were fed an average concentration of $0.5 \mu \mathrm{g}$ of imidacloprid per g tropical milkweed leaf for $24 \mathrm{~h}$. This concentration was obtained by applying 100 or $150 \mu \mathrm{L}$ of a $0.01 \mu \mathrm{g} / \mu \mathrm{L}$ imidacloprid suspension on approximately 2 or $3 \mathrm{~g}$ of leaf material, respectively. Leaves were air-dried and photographs of leaves were taken prior to larval feeding and $24 \mathrm{~h}$ after feeding to obtain the surface area consumed by each larva, similar to methods described in ${ }^{14}$. Based on the estimated leaf concentration and surface area of leaf consumed, an oral imidacloprid dose consumed by each larva was estimated (the use of silwet ensured that one side of a leaf surface was entirely coated with the imidacloprid suspension). Five to six larval/pupal samples, with each sample corresponding to a minimum of $700 \mathrm{mg}$ at the start of an experiment, were collected at various points (see Table S4). All larval/pupal samples were stored individually in plastic containers at $-20^{\circ} \mathrm{C}$ until analysis for concentrations of imidacloprid and its two metabolites (see analytical methodology in "Imidacloprid toxicokinetics in AE-susceptible and unsusceptible species" section). 
Observational details on pupation in corn earworms. Two-day old sixth instar corn earworms were treated with acetone and $20 \mu \mathrm{g}$ imidacloprid (one $\mu \mathrm{L}$ of a $20 \mu \mathrm{g} / \mu \mathrm{L}$ solution) and observed continually over three days (except from 20:00 to 06:00) for initiation of apolysis, denoted by HCS as observed under a dissecting microscope, and the beginning of ecdysis, denoted by commencement of tracheal shedding at the posterior end. This information was obtained for 24 larvae treated with acetone or imidacloprid $(\mathrm{n}=12 \mathrm{each})$. Time to landmark events were recorded.

Statistical analyses. All statistical analyses were done in RStudio 1.4.1106 (R version 4.0.4) ${ }^{41}$. Data for all species were analyzed independently and figures were made using "ggplot2" 2 . To identify if there was a linear relationship between percent $\mathrm{AE}$ and imidacloprid doses or times of treatment, Pearson's correlation test was performed and $\mathrm{R}$ (correlation coefficient) and $p$ (significance) values were obtained. A poisson glm model and type 3 ANOVA (obtained from the "car" package ${ }^{43}$ ) were used to evaluate differences in days to pupation/ AE between control and imidacloprid-treated larvae. Type 3 ANOVA was used to analyze differences in internal imidacloprid concentrations over time in monarch butterfly and fall armyworm larvae; if significance was obtained ( $p<0.05$ ), Dunnett's test for multiple comparisons ("emmeans" package ${ }^{44}$ ) was employed to compare baseline concentrations $(0-\mathrm{h})$ with concentrations measured at other time points. A Welch two sample t-test was used to analyze differences in the number of hours it took larvae in control and imidacloprid-treated groups to initiate pupal ecdysis following head capsule slippage.

\section{Data availability}

Data and metadata pertaining to this manuscript are publicly available at this GitHub repository: https://github. com/Niranjana296/Arrested-pupal-ecdysis.

Received: 20 February 2021; Accepted: 21 July 2021

Published online: 04 August 2021

\section{References}

1. Douglas, M. R. \& Tooker, J. F. Large-scale deployment of seed treatments has driven rapid increase in use of neonicotinoid insecticides and preemptive pest management in U.S. field crops. Environ. Sci. Technol. 49, 5088-5097 (2015).

2. U.S. Geological Survey. Estimated annual agricultural pesticide use maps. https://water.usgs.gov/nawqa/pnsp/usage/maps/ (2017).

3. U.S. Environmental Protection Agency. EPA actions to protect pollinators. https://www.epa.gov/pollinator-protection/epa-actio ns-protect-pollinators (2020).

4. Basley, K. \& Goulson, D. Effects of field-relevant concentrations of clothianidin on larval development of the butterfly Polyommatus icarus (Lepidoptera, Lycaenidae). Environ. Sci. Technol. 52, 3990-3996 (2018).

5. Halsch, C. A. et al. Pesticide contamination of milkweeds across the agricultural, urban, and open spaces of low-elevation northern California. Front. Ecol. Evol. 8, 162 (2020).

6. Olaya-Arenas, P. \& Kaplan, I. Quantifying pesticide exposure risk for monarch caterpillars on milkweeds bordering agricultural land. Front. Ecol. Evol. 7, 223 (2019).

7. Peterson, E. M., Shaw, K. R. \& Smith, P. N. Toxicity of agrochemicals among larval painted lady butterflies (Vanessa cardui). Environ. Toxicol. Chem. 38, 2629-2636 (2019).

8. Van Timmeren, S., Wise, J. C. \& Isaacs, R. Soil application of neonicotinoid insecticides for control of insect pests in wine grape vineyards. Pest Manag. Sci. 68, 537-542 (2012).

9. Christen, V., Mittner, F. \& Fent, K. Molecular effects of neonicotinoids in honey bees (Apis Mellifera). Environ. Sci. Technol. 50, 4071-4081 (2016).

10. Ankley, G. T. et al. Adverse outcome pathways: A conceptual framework to support ecotoxicology research and risk assessment. Environ. Toxicol. Chem. 29, 730-741 (2010).

11. LaLone, C. A. et al. Weight of evidence evaluation of a network of adverse outcome pathways linking activation of the nicotinic acetylcholine receptor in honey bees to colony death. Sci. Total Environ. 584-585, 751-775 (2017).

12. Camp, A. A. \& Lehmann, D. M. Impacts of neonicotinoids on the bumble bees Bombus terrestris and Bombus impatiens examined through an adverse outcome pathway framework lens. Environ. Toxicol. Chem. https://doi.org/10.1002/etc.4939 (2020).

13. Žitňan, D. \& Adams, M. E. Neuroendocrine regulation of ecdysis. In Insect endocrinology (ed. Lawrence, I. G.) 253-309 (Academic Press, 2012).

14. Krishnan, N. et al. Assessing field-scale risks of foliar insecticide applications to monarch butterfly (Danaus Plexippus) larvae. Environ. Toxicol. Chem. 39, 923-941 (2020).

15. Krishnan, N. et al. Monarch Butterfly (Danaus plexippus) life stage risks from foliar and seed-treatment insecticides. Environ. Toxicol. Chem. https://doi.org/10.1002/etc.5016 (2021).

16. Kim, Y. J. et al. Central peptidergic ensembles associated with organization of an innate behavior. Proc. Natl. Acad. Sci. U. S. A. 103, 14211-14216 (2006).

17. Bargar, T. A., Hladik, M. L. \& Daniels, J. C. Uptake and toxicity of clothianidin to monarch butterflies from milkweed consumption. PeerJ 8, e8669 (2020).

18. Heneberg, P., Bogusch, P., Astapenková, A. \& Řezáč, M. Neonicotinoid insecticides hinder the pupation and metamorphosis into adults in a crabronid wasp. Sci. Rep. 10, 7077 (2020).

19. Park, J. H., Schroeder, A. J., Helfrich-Förster, C., Jackson, F. R. \& Ewer, J. Targeted ablation of CCAP neuropeptide-containing neurons of Drosophila causes specific defects in execution and circadian timing of ecdysis behavior. Development 130, 2645-2656 (2003).

20. Veverytsa, L. \& Allan, D. W. Temporally tuned neuronal differentiation supports the functional remodeling of a neuronal network in Drosophila. Proc. Natl. Acad. Sci. U. S. A. 109, E748-E756 (2012).

21. Arakane, Y. et al. Functional analysis of four neuropeptides, EH, ETH, CCAP and bursicon, and their receptors in adult ecdysis behavior of the red flour beetle, Tribolium castaneum. Mech. Dev. 125, 984-995 (2008).

22. Lahr, E. C., Dean, D. \& Ewer, J. Genetic analysis of ecdysis behavior in drosophila reveals partially overlapping functions of two unrelated neuropeptides. J. Neurosci. 32, 6819-6829 (2012).

23. Lee, D., Orchard, I. \& Lange, A. B. Evidence for a conserved CCAP-signaling pathway controlling ecdysis in a hemimetabolous insect, Rhodnius prolixus. Front. Neurosci. 7, 207 (2013).

24. Žitňan, D. \& Daubnerová, I. Chapter 69-Crustacean cardioactive peptide. In Handbook of Hormones (eds Takei, Y. et al.) 442-442 (Academic Press, 2016). 
25. Davis, N. T., Homberg, U., Dircksen, H., Levine, R. B. \& Hildebrand, J. G. Crustacean cardioactive peptide-immunoreactive neurons in the hawkmoth Manduca sexta and changes in their immunoreactivity during postembryonic development. J. Comp. Neurol. 338, 612-627 (1993).

26. Song, Y., Villeneuve, D. L., Toyota, K., Iguchi, T. \& Tollefsen, K. E. Ecdysone receptor agonism leading to lethal molting disruption in arthropods: Review and adverse outcome pathway development. Environ. Sci. Technol. 51, 4142-4157 (2017).

27. Žitňan, D. \& Adams, M. E. Neuroendocrine regulation of insect ecdysis. In Comprehensive Molecular Insect Science Vol. 3 (eds Gilbert, L. I. et al.) 1-60 (Elsevier, 2005).

28. Ewer, J. Behavioral actions of neuropeptides in invertebrates: Insights from Drosophila. Horm. Behav. 48, 418-429 (2005).

29. Vömel, M. \& Wegener, C. Neurotransmitter-induced changes in the intracellular calcium concentration suggest a differential central modulation of CCAP neuron subsets in Drosophila. Dev. Neurobiol. 67, 792-808 (2007).

30. Zayas, R. M., Qazi, S., Morton, D. B. \& Trimmer, B. A. Neurons involved in nitric oxide-mediated cgmp signaling in the tobacco hornworm, Manduca sexta. J. Comp. Neurol. 419, 422-438 (2000).

31. Gammie, S. C. \& Truman, J. W. Neuropeptide hierarchies and the activation of sequential motor behaviors in the hawkmoth, Manduca sexta. J. Neurosci. 17, 4389-4397 (1997).

32. Zayas, R. M., Qazi, S., Morton, D. B. \& Trimmer, B. A. Nicotinic-acetylcholine receptors are functionally coupled to the nitric oxide/cgmp-pathway in insect neurons: Nicotinic evoked NO/cGMP-signaling. J. Neurochem. 83, 421-431 (2002).

33. Mannai, S., Bitri, L. \& Thany, S. H. CGMP/CGMP-dependent protein kinase pathway modulates nicotine-induced currents through the activation of a-bungarotoxin-insensitive nicotinic acetylcholine receptors from insect neurosecretory cells. J. Neurochem. 137, 931-938 (2016).

34. Žitňan, D. \& Adams, M. E. Excitatory and inhibitory roles of central ganglia in initiation of the insect ecdysis behavioural sequence. J. Exp. Biol. 203, 1329-1340 (2000).

35. Fuse, M. \& Truman, J. W. Modulation of ecdysis in the moth Manduca sexta: The roles of the suboesophageal and thoracic ganglia. J. Exp. Biol. 205, 1047-1058 (2002).

36. Mena, W., Diegelmann, S., Wegener, C. \& Ewer, J. Stereotyped responses of Drosophila peptidergic neuronal ensemble depend on downstream neuromodulators. Elife 5, e19686 (2016).

37. Brindley, T. A., Sparks, A. N., Showers, W. B. \& Guthrie, W. D. Recent research advances on the European corn borer in North America. Ann. Rev. Entomol. 20, 221-239 (1975).

38. Guthrie, W. D. Advances in rearing the European corn borer on a meridic diet. Proceedings of the International symposium on methodologies for developing host plant resistance to maize insects. CIMMYT, Mexico (1989).

39. Lewis, L. C. \& Lynch, R. E. Rearing the European corn borer, Ostrinia nubilalis, on diets containing corn leaf and wheat germ, Iowa State. J. Sci. 44, 9-14 (1969)

40. Suchail, S., Guez, D. \& Belzunces, L. P. Discrepancy between acute and chronic toxicity induced by imidacloprid and its metabolites in Apis mellifera. Environ. Toxicol. Chem. 20, 2482-2486 (2001).

41. R Core Team. R: A Language and Environment for Statistical Computing (R Foundation for Statistical Computing, 2021). https:// www.R-project.org/

42. Wickham, H. ggplot2: Elegant Graphics for Data Analysis (Springer, 2016).

43. Fox, J. \& Weisberg, S. An \{R\} Companion to Applied Regression 3rd edn (Sage, 2019). https://socialsciences.mcmaster.ca/jfox/Books/ Companion/

44. Length, R. V. emmeans: Estimated Marginal Means, Aka Least-Squares Means. R package Version 1.6.0 (2021).https://CRAN.R-proje ct.org/package $=$ emmeans

\section{Acknowledgements}

This research was funded by the Henry and Sylvia Richardson Research Incentive Grant obtained from the entomology department at Iowa State University (ISU) (\$1000 student grant). It was also supported in part by the Agriculture and Food Research Initiative Pollinator Health Program (Grant No. 2018-67013-27541) from the U.S. Department of Agriculture (USDA) National Institute of Food and Agriculture and State of Iowa funds. Keith Bidne, Corn Insects and Crop Genetics Research Unit (CICGRU), USDA, Ames, Iowa, provided monarch butterfly and European corn borer larvae. Royce Bitzer, CICGRU-USDA, Ames, Iowa, helped capture red admiral butterflies, and assisted with the rearing of red admiral and painted lady larvae. Abigail Kropf, graduate student in entomology at ISU, shared techniques for rearing wax moth larvae. Taylor Boysen, undergraduate at ISU, helped conduct toxicity bioassays. Laura Burns and Dwayne Schrunk in the ISU College of Veterinary Medicine, Veterinary Diagnostic Laboratory, quantified imidacloprid concentrations in monarch butterfly and fall armyworm larvae. Mention of trade names or commercial products in this publication is solely for the purpose of providing specific information and does not imply recommendation or endorsement by ISU.

\section{Author contributions}

N.K. and R.A.J. conducted the experiments. N.K. wrote the first draft of the manuscript. All authors were involved in conceptualizing the study and editing the manuscript.

\section{Competing interests}

The authors declare no competing interests.

\section{Additional information}

Supplementary Information The online version contains supplementary material available at https://doi.org/ 10.1038/s41598-021-95284-0.

Correspondence and requests for materials should be addressed to N.K.

Reprints and permissions information is available at www.nature.com/reprints.

Publisher's note Springer Nature remains neutral with regard to jurisdictional claims in published maps and institutional affiliations. 
(c) (i) Open Access This article is licensed under a Creative Commons Attribution 4.0 International cc) License, which permits use, sharing, adaptation, distribution and reproduction in any medium or format, as long as you give appropriate credit to the original author(s) and the source, provide a link to the Creative Commons licence, and indicate if changes were made. The images or other third party material in this article are included in the article's Creative Commons licence, unless indicated otherwise in a credit line to the material. If material is not included in the article's Creative Commons licence and your intended use is not permitted by statutory regulation or exceeds the permitted use, you will need to obtain permission directly from the copyright holder. To view a copy of this licence, visit http://creativecommons.org/licenses/by/4.0/.

(C) The Author(s) 2021 